\title{
UNIVERSALLY INCOMPARABLE RING-HOMOMORPHISMS
}

\author{
David E. Dobbs and Marco Fontana
}

A homomorphism $f: R \rightarrow T$ of (commutative) rings is said to be universally incomparable in case each base change $R \rightarrow S$ induces an incomparable map $S \rightarrow S \otimes_{R} T$. The most natural examples of universally incomparable homomorphisms are the integral maps and radiciel maps. It is proved that a homomorphism $f: R \rightarrow T$ is universally incomparable if and only if $f$ is an incomparable map which induces algebraic field extensions of fibres, $k\left(f^{-1}(Q)\right) \rightarrow k(Q)$, for each prime ideal $Q$ of $T$. In several cases ( $f$ algebra-finite, $T$ generated as $R$-algebra by primitive elements, $T$ an overring of a one-dimensional Noetherian domain $R$ ), each universally incomparable map is shown to factor as a composite of an integral map and a special kind of radiciel.

\section{Introduction}

Considerable attention has been paid over the years to the properties of lying-over, going-up, going-down, and incomparability concerning the behavior of prime ideals relative to homomorphisms, especially inclusion maps, of commutative rings ( $c f .[12$, page 28]). It has seemed natural to

Received 14 lNovember 1983. The first author was supported in part by grants from the University of Tennessee, Faculty Development Program and the Università di Roma. The second author's work was done under the Auspices of the GirsAGA of the CNR.

Copyright Clearance Centre, Inc. Serial-fee code: 0004-9727/84 $\$ A 2.00+0.00$. 
consider as well the corresponding universal properties, and most of these have been characterized. (As usual, if $P$ is a property of homomorphisms, then $R \rightarrow T$ is said to be universally $P$ in case, for each change of base $R \rightarrow S$, the induced homomorphism $S \rightarrow S \Theta_{R} T$ satisfies $\left.P.\right)$ Indeed, universally lying-over is equivalent to lying-over [11, Proposition 3.6.1 (ii), page 244]; universally going-up is equivalent to integra] [2, Lemma, page 160]; and universally going-down has been closely linked in [4] with the notion of weak normalization, in the sense of Andreotti and Bombieri. Our purpose here is to complete this circle of ideas by studying the universally incomparable homomorphisms. These maps are characterized first in Theorem 2.2 in ways that are, perhaps surprisingly, not wholly analogous to characterizations of related classes of homomorphisms in [4], [13].

The most natural examples of universally incomparable homomorphisms are the integral maps and the radiciel maps. (As in [11], a homomorphism $f: R \rightarrow T$ is said to be radiciel in case the induced function $f^{*}: \operatorname{Spec}(T) \rightarrow \operatorname{Spec}(R)$ is an injection and whenever $f^{*}(q)=p$, the resulting field extension $R_{p} / p R_{p} \rightarrow T_{q} / q T_{q}$ is purely inseparable. As radiciel is equivalent to universally radiciel [11, Proposition 3.7.1, page 246], it evidently implies universally incomparable.) since any composition of incomparable maps is incomparable and since tensor product commutes with direct limit, any composition of universally incomparable maps is universally incomparable. A sharp converse is available in the algebra-finite case, for then any universally incomparable map is the composite of an integral map with a special type of radiciel homomorphism (Remark $2.12(b)$ ). Moreover, each incomparable map is universally incomparable in the algebra-finite case (Corollary 2.7), but not in general (Remark 2.3). Factoring results of the above sort are also obtained for two types of maps $R+T$ which need not be algebra-finite. In the first of these (Corollary 2.8, Remark 2.12(c)), $T$ is assumed to be generated as an $R$-algebra by algebraic elements of a particular kind. The second (Proposition 2.13) addresses a Noetherian context and leads to a geometric example (Example 2.14) illustrating that universally incomparable overring extensions form a strictly larger class than the universally going-down overring extensions characterized in [4].

Throughout, all rings are assumed commutative, with unit; and all 
ring-homomorphisms are assumed unital. In addition, $x=x_{1}, \ldots, x_{n}$ denote commuting algebraically independent indeterminates over the appropriate rings. If $f: R \rightarrow T$ is a ring-homomorphism, then $f_{n}$ denotes the induced homomorphism $R\left[X_{1}, \ldots, X_{n}\right] \rightarrow T\left[X_{1}, \ldots, X_{n}\right] ;$ and if $p$ is a prime of $R$, then $k_{R}(p)$ denotes $R_{p} / p R_{p}$ and $T_{p}$ denotes $T_{f(R \backslash p)}\left(\cong T \otimes_{R} R_{p}\right)$. Any unexplained material is standard, as in [9] and [12].

\section{Results}

Before characterizing universally incomparable homomorphisms, we begin by adapting some material from [13]. Let $A$ be a commutative ring and let $p \in \operatorname{Spec}(A)$. The prime ideal $p A[X]$ of the polynomial ring $A[X]$ will be denoted by $p^{*}$. For each monic irreducible $\alpha \in k_{A}(p)[X]$, the upper of $p$ corresponding to $\alpha$ is $\langle p, \alpha\rangle=\{h \in A[X] \mid$ the canonical image of $h$ in

$(A / p)[X]$ is divisible by $\alpha\}$.

The following result was obtained by McAdam [13, Theorems 1 and 2] for the case of inclusion maps of domains, but the methods of [13] carry over directly, and so we omit the proof:

LEMMA 2.1. Let $R$ be a ring and $p$ a prime ideal of $R$. Then:

(a) Let $j: R \rightarrow R[X]$ be the canonical inclusion map. Then the prime ideals $P$ of $R[X]$ such that $j^{-1}(P)=p$ are of two types: $p^{*}$ and the uppers of $p$. Moreover, $p$ is properly contained in each upper of $p$; and if $\alpha, \gamma$ are distinct monic irreducible polynomials in $k_{R}(p)[X]$, then $\langle p, \alpha\rangle$ and $\langle p, \gamma\rangle$ are incomparable.

(b) Let $f: R \rightarrow T$ be a ring-homomorphism, with $f_{1}: R[X] \rightarrow T[X]$ the induced homomorphism. Then for each monic irreducible $\alpha \in k_{R}(p)[X]$, the primes $Q$ of $T[X]$ such that $f_{1}^{-1}(Q)=\langle p, \alpha\rangle$ are the uppers of the form $(q, B)$, where $q \in \operatorname{spec}(T), f^{-1}(q)=p$, and the canonical inclusion $k_{R}(p)[X] \rightarrow k_{T}(q)[X]$ sends $\alpha$ to a polynomial which is 
divisible by $B$. Moreover, the primes $Q$ of $T[X]$ such that $f_{1}^{-1}(Q)=p^{*}$ are of two types: $q^{*}$ and $\langle q, \delta\rangle$, where $q \in \operatorname{Spec}(T)$, $f^{-1}(q)=p$, and no monic imeducible polynomial in $k_{R}(p)[X]$ is divisible by $\delta$.

The following definition will be of fundamental importance. A ringhomomorphism $f: R \rightarrow T$ is called residually algebraic if, for each $q \in \operatorname{Spec}(T)$ and $p=f^{-1}(q)$, the induced field extension $k_{R}(p) \rightarrow k_{T}(q)$ is algebraic. The role of this property in the following characterization of "universally incomparable" should be contrasted with the part played by purely inseparable requirements in characterizations of radiciel and various related properties [4, Theorems 2.1 and 2.5] (cf. also [13, Theorems 3 and $B]$ ).

THEOREM 2.2. For a ring-homomoxphism $f: R+T$, the following conditions are equivalent:

(i) $f$ is universally incomparable;

(ii) $f$ is incomparable and residually algebraic;

(iii) the inclusion map $f(R) \rightarrow T$ is universally incomparable;

(iv) there exists $n \geq 1$ such that $f_{n}$ is incomparable;

(v) $f_{1}$ is incomparabie;

(vi) for each $n \geq 0, f_{n}$ is incomparabie;

(vii) for each $n \geq 0, f_{n}$ is incomparable and residually algebraic.

Proof. It is evident that $(v i i) \Rightarrow(v i) \Rightarrow(v) \Rightarrow(i v)$.

$(i) \Rightarrow$ (vi). Trivial since $R\left[X_{1}, \ldots, X_{n}\right] \otimes_{R} T \cong T\left[X_{1}, \ldots, X_{n}\right]$.

$(v i) \Rightarrow(i)$. It is known that direct limit preserves incomparability [6, Proposition 2.3]. Consequently, the criterion in [4, Proposition 2.2] reduces the present assertion to the easy observation that if $f$ is incomparable, so is the induced map $R / J \rightarrow T / J T$ for each ideal $J$ of $R$. 
(i) $\Leftrightarrow$ (iii). $f_{n}$ is incomparable if and only if the inclusion map $f(R)\left[X_{1}, \ldots, X_{n}\right]+T\left[X_{1}, \ldots, X_{n}\right]$ is incomparable, because $f(R)\left[X_{1}, \ldots, X_{n}\right]$ is the image of $f_{n}$. The present assertion therefore follows since we have already seen that $(i) \Leftrightarrow(v i)$.

The remainder of the proof will sketch how to modify various arguments of McAdam [13], originally used to treat (what [4, Theorem 2.5] showed to be) universally unibranched inclusion maps of domains.

(v) $\Rightarrow$ (ii). Assume (v). That $f$ is incomparable now follows from Lemma $2.1(a)$ and the fact that $f^{-1}(q)=p$ entails $f_{1}^{-1}\left(q^{*}\right)=p^{*}$. In view of Lemma 2.1 (b), we can show that $f$ is residually algebraic by reasoning as in [13, page 709,1 ines $4-8]$.

$(i i) \Rightarrow(v)$. Assume (ii). If $(v)$ fails, there exist distinct primes $Q_{1} \subset Q_{2}$ of $T[X]$ such that $f_{1}^{-1}\left(Q_{1}\right)=f_{1}^{-1}\left(Q_{2}\right)=P \in \operatorname{Spec}(R[X])$. If $P=\langle p, \alpha\rangle$ then Lemma 2.1 gives $Q_{i}=\left\langle q_{i}, \gamma_{i}\right\rangle$, where $f^{-1}\left(q_{i}\right)=p$ and $q_{1} \neq q_{2}$. As $q_{i}$ lies over $q_{i}$, it follows that $q_{1} \subset q_{2}$, contradicting incomparability of $f$. The remaining case, in which $P$ assumes the form $p^{*}$, may be treated as in [13, page 709, lines 14-18].

$(i v) \Rightarrow(v)$. Since $\left(f_{n-1}\right)_{1}=f_{n}$, the present assertion follows from the first observation in the above proof that $(v) \Rightarrow(i i)$.

(vi) $\Rightarrow$ (vii). Since $\left(f_{n}\right)_{1}=f_{n+1}$, the present assertion follows since we have already seen that $(v) \Rightarrow(i i)$.

$(v) \Rightarrow(v i)$. Since $f_{n+2}=\left(f_{n}\right)_{2}$ and since $(v) \Leftrightarrow(i i)$, it is enough to show that $(i i)$ implies $f_{1}$ is residually algebraic. Accordingly, assume (ii), and consider $Q \in \operatorname{Spec}(T[X])$, with $P=f_{1}^{-1}(Q)$. If $Q=q^{*}$ then $P=p^{*}$ with $p=f^{-1}(q)$, and the asserted algebraicity of $k_{R[X]}(P)=k_{R}(p)(X) \rightarrow k_{T[X]}(Q)=k_{T}(q)(X)$ follows from the assumed algebraicity of $k_{R}(p) \rightarrow k_{T}(q)$. In view of Lemma $2.1(b)$, the residually algebraic nature of $f$ readily assures that the only remaining case is 
$Q=\langle q, B\rangle, \quad P=\langle p, \alpha\rangle$. By taking suitable localizations of factorrings, precisely as in [13, page 710], we need only prove the following statement. If $K_{1} \subset K_{2}$ is an algebraic field extension and $P_{i}$ is a maximal ideal of $K_{i}[X]$, then the field extension $K_{1}[X] / P_{1} \rightarrow K_{2}[X] / P_{2}$ is algebraic. This, however, is evident since algebraicity is transitive. The proof is complete.

REMARK 2.3. An incomparable ring-homomorphism need not be universally incomparable. Surely the simplest example of this is a transcendental field extension $k \rightarrow k(Y)$, for it is evidently not residually algebraic. Of course, $k(Y)$ is not algebra-finite over $k$ (by, for instance, Hilbert's Nullstellensatz), thus suggesting the positive result in Corollary 2.7 below.

The following definition will be helpful. If $f: R \rightarrow T$ is a ringhomomorphism and $p \in \operatorname{Spec}(R)$, then $T(p)=T(p ; f)$ will denote $T \otimes_{R} k_{R}(p)\left[\cong T_{p} / p T_{p}\right]$. Notice that $\operatorname{Spec}(T(p))$ is isomorphic, qua topological space or partially ordered set, with

$$
\left\{q \in \operatorname{Spec}(T) \mid f^{-1}(q)=p\right\} \text {. }
$$

When the latter set is empty, $T(p)$ is the zero-ring, whose dimension is conventionally taken as -1 .

The $T(p)$ notation leads to the following characterization of incomparability. In view of the above comments, its proof may be left to the reader.

LEMMA 2.4. For a ming-homomorphism $f: R \rightarrow T$, the following conditions are equivalent:

(i) $f$ is incomparable;

(ii) for each $p \in \operatorname{Spec}(R)$ such that $T(p) \neq 0$, the canonical homomorphism $k_{R}(p) \rightarrow T(p)$ is incomparable;

(iii) for each $p \in \operatorname{Spec}(R), \operatorname{dim}(T(p)) \leq 0$;

(iv) for each $p \in \operatorname{Spec}(R)$ such that $T(p) \neq 0$, the induced map $R_{p} \rightarrow T_{p}$ is incomparable.

In view of Theorem $2.2[(i) \Leftrightarrow$ (ii)], we obtain characterizations of 
"universally incomparable" by augmenting each of the conditions in Lemna 2.4 with "for each $p \in \operatorname{Spec}(R)$ such that $T(p) \neq 0$, the canonical ringhomomorphism $k_{R}(p) \rightarrow T(p)$ is residually algebraic". Next, we tend to the zero-dimensional case, by showing that universally incomparable, in that context, implies integral.

PROPOSITION 2.5. Let $f: R \rightarrow T$ be a ring-homomorphism. Then:

(a) Let $R, T$ each be zero-dimensional mings. Then $f$ is integral if (and only if) $f$ is residually algebraic.

(b) Let $f$ appear in a pulzback diagram

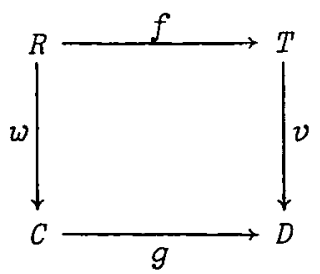

in which $C, D$ are zero-dimensional rings, $g$ is an inclusion, $T$ is quasilocal, and $v$ is surjective. Then $f$ is integral if (and only if) $f$ is residually algebraic.

Proof. (a) By passing to $f(R) \rightarrow T$, we may assume that $f$ is an inclusion map. If the result fails, select $b \in T$ such that $b$ is not integral over $R$. Then

$$
S=\left\{b^{n}+r_{1} b^{n-1}+\ldots+r_{n-1} b+r_{n} \mid r_{i} \in R \text { for each } i ; n \geq 1\right\} \cup\{1\}
$$

is a multiplicative subset of $T$ which does not contain 0 . Accordingly (cf. [12, Theorem 1]), $T$ has a prime ideal $N$ which is disjoint from $S$. Let $M=N \cap R$. As $N$ and $M$ are maximal ideals in $T$ and $R$, respectively, algebraicity of the field extension $R / M \rightarrow T / N$ produces an equation

$$
(b+N)^{n}+\left(r_{1}+N\right)(b+N)^{n-1}+\ldots+r_{n}+N=0 \in T / N
$$

for suitable $r_{i} \in R, n \geq I$. But then

$$
b^{n}+r_{1} b^{n-1}+\ldots+r_{n} \in N \cap S=\varnothing
$$

the desired contradiction. 
(b) It is well-known (and easy to see) that $I=\operatorname{ker}(v)$ coincides with $\operatorname{ker}(w)$, so that $D$ (respectively, $C$ ) may be identified with $T / I$ (respectively, $R / I$ ). One readily verifies then that $g$ inherits the residually algebraic property from $f$. Therefore, by $(a), g$ is integral. Applying [8, Proposition 2.2 (10)] to the above pull back diagram or calculating directly with the help of the above identifications, one shows that $f$ is integral, completing the proof.

We pause to record an application of Proposition 2.5 (b). Let $f: R \rightarrow T$ be an inclusion map of rings such that $\operatorname{Spec}(R)=\operatorname{Spec}(T)$ as sets (or, equivalently, as topological spaces). Then $f$ is integral if (and only if) $f$ is residually algebraic. (For the proof, we may assume $R \neq T$. Then $R$ is easily seen to be quasilocal, say with maximal ideal $M$, and we may apply Proposition $2.5(b)$ to the pullback description $\left.R=T \times T / M^{R / M} \cdot\right)$

It is interesting to note that "Spec $(R)=\operatorname{Spec}(T)$ as sets" is not a universal property. (Contrast the situation for schemes!) Indeed, let $K / F$ be a transcendental extension of distinct fields, $B=K|[X]|=K+M$ (with $M=X B$ ), and $A=E+M$. It is well-known (cf. [9, Exercise 12, page 202]) that $\operatorname{spec}(A)=\{0, M\}=\operatorname{spec}(B)$. However $k_{A}(M) \rightarrow k_{B}(M)$ is just $F \rightarrow K$, which is not algebraic. Thus, by Theorem 2.2 $[(i) \Longleftrightarrow(i i) \Longleftrightarrow(v)]$, the inclusion map $A[Y] \rightarrow B[Y]$ is not incomparable; a fortiori, $\operatorname{Spec}(A[Y]) \neq \operatorname{Spec}(B[Y])$.

We next consider the algebra-finite context.

PROPOSITION 2.6. Let $f: R \rightarrow T$ be an incomparable ringhomomorphism relative to which $T$ is algebra-finite over $R$ and $R$ is integrally closed in $T$. Then $f$ is radiciel. Moreover, if $q \in \operatorname{spec}(T)$ and $p=f^{-1}(q)$, then the canonical field extension $k_{R}(p) \rightarrow k_{T}(q)$ is an isomorphism.

Proof. By passing to $f(R) \rightarrow T$, we may assume that $f$ is an inclusion map. Let $q, p$ be as in the statement. Note that $R_{p}$ is integrally closed in $T_{p}$; by incomparability, $q T_{p}$ is isolated in its fibre (over $p R_{p}$ ); and $T_{p}$ is algebra-finite over $R_{p}$. Thus by Zariski's Main Theorem (as in, for instance, [7]), there exists an element 
$g \in R_{p} \backslash p R_{p}$ such that $\left(T_{p}\right)_{g}=\left(R_{p}\right)_{g}$. Since $g$ is a unit of $R_{p}$ (and of $\left.T_{p}\right)$, this entails $T_{p}=R_{p}$, whence $q T_{p}=p_{p}$. If $j: T \rightarrow T_{p}$ is the canonical homomorphism, it follows that $j^{-1}\left(p R_{p}\right)=q$. Hence the canonical function $\operatorname{Spec}(T)+\operatorname{Spec}(R)$ is an injection.

It suffices to prove the final assertion. For $q, p$ as above, the fact that $T_{p}=R_{p}$ combines with [9, Corollary 5.2] to yield $T_{p} \cong T_{q}$. Thus we obtain the canonical identifications

$$
k_{T}(q)=T_{q} / q T_{q}=T_{p} / q T_{p}=R_{p} / p R_{p}=k_{R}(p),
$$

completing the proof.

COROLLARY 2.7. Let $f: R \rightarrow T$ be a ring-homomorphism relative to which $I$ is algebra-finite over $R$. Then $f$ is universally incomparable if (and only if) $f$ is incomparable.

Proof. Let $S$ be the integral closure of $R$ in $T$. Then $f=h g$, for the canonical homomorphisms $g: R \rightarrow S$ and $h: S \rightarrow T$. By integrality, $g$ is universally incomparable, and so it suffices to show that $h$ is universally incomparable. However this, in turn, follows from Proposition 2.6 since $h$ inherits incomparability from $f$. The proof is complete.

The preceding ideas permit us next to give another class of examples of universally incomparable homomorphisms. Following [3], we shall say that an element $b$ of a commutative $R$-algebra $T$ is primitive over $R$ in case $g(b)=0$ for some $g \in R[X]$ with at least one coefficient equal to 1 .

COROLLARY 2.8. Let $f: R \rightarrow T$ be a ring-homomorphism relative to which $T$ may be generated as an R-algebra by a set $S=\left\{b_{i}\right\}$, where each $b_{i}$ is primitive over $R$. Then $f$ is voniversally incomparable. Moreover, if $R$ is integrally closed in $T$, then $f$ is radiciel.

Proof. By Theorem $2.2[(i i i) \Rightarrow(i)]$, we may assume that $f$ is an inclusion map. In addition, we may assume that $T$ is algebra-finite over $R$. (The points involved are that $T=\underset{\lim }{\longrightarrow}\left[b_{1}, \ldots, b_{n}\right]$ where $\left\{b_{1}, \ldots, b_{n}\right\}$ ranges over the finite subsets of $s ;$ and direct limit 
preserves (universal) incomparability [6, Proposition 2.3].) Thus, we can suppose that $s=\left\{b_{1}, \ldots, b_{n}\right\}$, so that $f$ "factors" as

$$
R \subset R\left[b_{1}\right] \subset R\left[b_{1}, b_{2}\right] \subset \ldots \subset R\left[b_{1}, b_{2}, \ldots, b_{n-1}\right] \subset R\left[b_{1}, \ldots, b_{n}\right\rceil=T .
$$

However for each $i \geq 0, b_{i+1}$ is primitive over $R\left[b_{1}, \ldots, b_{i}\right]$, and so [3, Theorem] assures that the extension $\left.R\left[b_{1}, \ldots, b_{i}\right] \subset R \mid b_{1}, \ldots, b_{i+1}\right]$ is incomparable. By considering the displayed tower, we thus see that $f$ is incomparable, and so an application of Corollary 2.7 establishes the first assertion. Using the fact that direct limit preserves radiciel [4, Lemma 2.4 (b)], and appealing to Proposition 2.6 instead of Corollary 2.7 , one may fashion a parallel proof of the second assertion.

REMARK 2.9. The hypothesis of Corollary 2.8 was suggested by a characterization of integrality in [3, Remark 8 (c)]. We next record a nonintegral application of Corollary 2.8: if $T$ is an overring of a Prüfer domain $R$, then the inclusion map $f: R \rightarrow T$ is radiciel. Indeed, each element of $T$ is primitive over $R$ (by either [10, Theorem 2] or [3, Corollary 5]) and $R$ is integrally closed (in $T$ ), so that the second assertion in Corollary 2.8 yields the desired conclusion.

Another proof of the result in the preceding paragraph uses ideas that will reappear in the proof of Proposition 2.10. To wit: since $f$ is flat, it is a universally going-down overring extension, and hence is radiciel by $[4$, Theorem 3.17 and Corollary 3.12 (b)].

Let $P$ be a property of (some) ring-homomorphisms. Following [5], we shall say that a domain $R$ is a $P$-domain in case the inclusion map $R \rightarrow T$ satisfies $P$ for each overring $T$ of $R$.

PROPOSITION 2.10. For an integrally closed domain $R$, the following conditions are equivalent:

(i) $R$ is a coniversally incomparable-domain;

(ii) $R$ is a radiciel-domain;

(iii) $R$ is a universalzy going-down-domain;

(iv) $R$ is a Prüfer domain.

Proof. $(i i) \Rightarrow(i)$. Trivial.

(iii) $\Leftrightarrow(i v)$. This is [5, Corollary 2.3]. 
$(i) \Rightarrow(i v)$. It is well-known that an integrally closed incomparabledomain must be a Prüfer domain (cf. [9, Theorem 26.2]).

$(i i i) \Rightarrow(i i)$. Assume $(i i i)$, and consider the inclusion map $f: R \rightarrow T$ for an overring $T$ of $R$. Since $f$ is a universally going-down overring map, [4, Theorem 3.17] implies that $f$ satisfies the UGD property and so, by $[4$, Corollary $3.12(\mathrm{~b})], f$ is radiciel, completing the proof.

REMARK 2.11. One may, in the spirit of [5], proceed to develop a theory for universally incomparable-domains. Typical results state that a domain $R$ is a universally incomparable-domain if and only if each localization $R_{M}$ is; and, as above, each universally going-down-domain is a universally incomparable-domain. However, such domains seem less fruitful than the universally going-down domains, since they fail to sustain the analogue of [5, Theorem 2.6]. Indeed, there is no (universally incomparable-) domain $R$ for which the inclusion map $R \rightarrow T$ is (universally) incomparable for each domain $T$ containing $R:$ consider $T=R[X]$ !

We next formalize a concept which has appeared implicitly in Proposition 2.6 and Corollary 2.8. A (typically injective) ringhomomorphism $f: R \rightarrow T$ will be called an essential-identity (and $R, T$ will be called essentially equal) if, for each $p \in \operatorname{Spec}(R)$ such that $T(p) \neq 0$, the induced map $R_{p} \rightarrow T_{p}$ is an isomorphism. The next remark collects some relevant material.

REMARK 2.12. (a) Let $f$ be an essential-identity. Then Lemma 2.4 $[(i v) \Rightarrow(i)]$ implies that $f$ is incomparable. In fact, $f$ is universally incomparable since, by the latter part of the proof of Proposition 2.6, $f$ is actually radiciel.

(b) By (a), the combined effect of Proposition 2.6 and Corollary 2.7 is the following assertion. Let $f: R \rightarrow T$ be an incomparable ringhomomorphism relative to which $T$ is algebra-finite over $R$. Let $S$ be the integral closure of $R$ in $T$, and consider the canonical homomorphisms $g: R \rightarrow S$ and $h: S \rightarrow T$. Then $f=h g$ is universally incomparable since $g$ is integral and $h$ is an essential-identity.

(c) It is easy to verify (using [11, Propositions 6.1.2-6.1.6, pages 128-130 ]) that direct limit preserves the essential-identity property. Thus, by (b), the second assertion of Corollary 2.8 may be strengthened, in 
case $f$ is an injection, to say that $R$ and $T$ are essentially equal.

(d) The characterization of flat overrings in [14, Theorem 1] readily leads to the following result. Let $T$ be an overring of a domain $R$, with $f: R \rightarrow T$ the inclusion map. If $T$ is $R$-flat, then $f$ is an essential-identity; the converse holds if $\operatorname{dim}(R)=1$.

In the absence of finite-type hypotheses, a factoring result in the spirit of Remark 2.12 (b) seems unavailable. However, we do have the following important special case.

PROPOSITION 2.13. Let $T$ be an overring of a one-dimensional Noetherian domain $R$, with $f: R \rightarrow T$ the inclusion map. Then $f$ is coniversally incomparable. Indeed, if $S$ denotes the integral closure of $R$ in $T$, with $g: R \rightarrow S$ and $h: S \rightarrow T$ the canonical maps, then $f=h g, g$ is integral, and $h$ is flat. (Hence $h$ is also an essential-identity and radiciel.)

Proof. The first assertion may be seen directly since the KrullAkizuki Theorem, in the version given in [1, Proposition 5, page 500], readily implies that $f$ satisfies condition ( $i i$ ) in the statement of Theorem 2.2.

We may therefore assume that $R=S$ is integrally closed in $T$. By Remark 2.12(a) and (d), it is enough to prove that $f$ is an essentialidentity. Consider $M \in \operatorname{Spec}(R)$ such that $T(M) \neq 0$; we must show that $R_{M} \rightarrow T_{M}$ is an isomorphism. Without loss of generality, $M \neq 0$. By abus de langage, we may further assume that $(R, M)$ is quasi-local, and need to show that $f$ is surjective.

It is enough to prove that $R$ contains each element $u \in T$. Set $A=R[u]$. Choose a nonzero prime $N$ of $T$. (Such exists since $T(M) \neq 0$. .) By the remarks made two paragraphs ago, $N \cap A$ is isolated in its fibre (above $M$ ). Then, just as in the proof of Proposition 2.6, Zariski's Main Theorem leads to $A=R$. This completes the proof.

One consequence of Proposition 2.13 is that each one-dimensional Noetherian domain $R$ is a universally incomparable-domain. In view of Remarks 2.11 and 2.9, it is important to note that such $R$ need not be a universalzy going-down-domain. In closing, we shall illustrate this fact with a geometric example. 
EXAMPLE 2.14. Let $R$ be the local ring at the origin for the nodal curve $y^{2}=x^{3}+x^{2}$. In other words, $R$ is the localization of $\mathrm{C}[X, Y] /\left(Y^{2}-X^{3}-X^{2}\right)$ at the canonical image of $(X, Y)$. Certainly, $R$ is a one-dimensional local (Noetherian) domain, say with maximal ideal $M$. Let $R^{\prime}$ be the integral closure of $R$; of course, $R^{\prime}$ has but two maximal ideals, say $N_{1}$ and $N_{2}$. As $R$ is seminormal with residue fields of characteristic zero, $R$ is the weak normalization (in the sense of Andreotti-Bombieri) of $R$ in $R^{\prime}$. So $R^{\prime}$ is not that weak normalization, whence [5, Theorem 2.4] assures that $R$ is not a universally-going-down domain. Accordingly, by [5, Theorem 2.6], $R$ has a valuation overring $T$ such that the inclusion map $f: R \rightarrow T$ is not universally going-down. However, $T=\left(R^{\prime}\right)_{N_{i}}$ and, as predicted by

Proposition 2.13, $f$ factors as the composite of the integral map $R \rightarrow R^{\prime}$ and the radiciel (essential-identity, flat) map $R^{\prime} \rightarrow T$.

\section{References}

[1] N. Bourbaki, Commutative algebra (Addison-Wesley, Reading, 1972).

[2] M. Demazure and P. Gabriel, Introduction to algebraic geometry and algebraic groups (North Holland, Amsterdam, 1980).

[3] D.E. Dobbs, "On INC-extensions and polynomials with unit content", Canad. Math. Buzz. 23 (1980), 37-42.

[4] D.E. Dobbs and M. Fontana, "Universally going-down homomorphisms of commutative rings", J. Algebra (to appear).

[5] D.E. Dobbs and M. Fontana, "Universally going-down integral domains", Arch. Math. (Basel) (to appear).

[6] D.E. Dobbs, M. Fontana, and 1.J. Papick, "Direct limits and goingdown", Comment. Math. Univ. St. Pauli 31 (1982), 129-135.

[7] E.G. Evans, Jr., "A generalization of Zariski's main theorem", Proc. Amer. Math. Soc. 26 (1970), 45-48.

[8] M. Fontana, "Topologically defined classes of commutative rings", Ann. Mat. Pura Appl. 123 (1980), 331-355. 
[9] R. Gilmer, Multiplicative ideal theory (Dekker, New York, 1972).

[10] R. Gilmer and J.F. Hoffman, "A characterization of Prüfer domains in terms of polynomials", Pacific J. Math. 60 (1975), 81-85.

[11] A. Grothendieck and J.A. Dieudonné, Eléments de géométrie algébrique, I (Springer-Verlag, Berlin, Heidelberg, New York, 1971).

[12] 1. Kaplansky, Commutative rings, revised edition (University of Chicago Press, Chicago, 1974).

[13] S. McAdam, "Going down in polynomial rings", Canad. J. Math. 23 (1971), 704-711.

[14] F. Richman, "Generalized quotient rings", Proc. Amer. Math. Soc. 16 (1965), 794-799.

Department of Mathematics,

University of Tennessee,

Knoxville,

Tennessee 37996,

USA;

Dipartimento di Matematica,

Università di Roma 1 ,

00185 Roma,

Italy. 